\title{
Vertical Axis Wind Turbine Case Study: Cost and Losses associated with Variable Torque and Variable Speed Strategies
}

\author{
M. Argent*, A.S. McDonald \\ University of Strathclyde, United Kingdom, *email: michael.argent@strath.ac.uk,
}

\begin{abstract}
Keywords: VAWT, Drivetrain, Torque Control, Generator

Abstract

This case study investigates the generator of a large offshore Vertical Axis Wind Turbine (VAWT). In particular this case study focuses on the effect of the inherent cyclic torque loading of the VAWT on the generator in terms of the costs and losses experienced. A spectrum of torque control strategies were created based on the ratio $q$ of the allowed electrical torque variation to the mechanical torque variation. Equations relating the copper and iron losses of the generator to this $q$ factor were established and investigated for a test turbine. In addition the relationship between the allowed variation in electrical torque and the cost of the active materials of the generator was explored.
\end{abstract}

\section{Introduction}

The drive to reduce the cost of energy of offshore generation has led to the development of large multi-MW Horizontal Axis Wind Turbines (HAWTs). In the offshore environment a greater premium is placed on increasing the availability of the turbine by improving reliability, accessibility and maintainability. With the turbines tens of $\mathrm{km}$ from shore and a majority of components elevated around $100 \mathrm{~m}$ above sea level this can cause issues.

Previous studies have been carried out into the application of Vertical Axis Wind Turbines (VAWTs) for onshore generation, in particular the NREL Darrieus machines of the 1980s [1] [2] although the project died out. Recently there has been interest in VAWTs for offshore generation both by academia and industry. These projects include the NOVA V-rotor Aerogenerator design [3] and the VertAx H-rotor design [4]. Potential benefits of VAWTs for offshore generation include the base mounted powertrain components (on most VAWT designs) allowing for easier access to components for maintenance and a lower centre of gravity which could potentially make them more suitable for floating platforms (trials of this are currently being conducted in France [5]). In addition, cyclic loading (self-weight) of HAWTs mean that increasing the size of HAWTs beyond 10MW might prove difficult, which could be an opportunity for VAWTs to take advantage of economies of scale [1][6].
While there has been notable research into aerodynamics and structural loading of VAWTs, there has been less research into the powertrains of VAWTs - the equipment used to convert mechanical power from the wind turbine rotor into useful electrical power - and the specific challenges that VAWTs present on the powertrain. The research into this does include Eriksson's Direct-Drive Permanent Magnet Generator for a $12 \mathrm{~kW}$ VAWT [7], the Musgrove VAWTs (up to $500 \mathrm{~kW}$ ) of the 1980's and 1990's [8], and Michon's feasibility study into Permanent Magnet Generators for the NOVA V-rotor VAWT [3].

One aspect of VAWTs is that they tend to have a lower rotational speed compared to an equivalent HAWT with the same rating - therefore the powertrain will have to be rated to a higher torque rating and may be more expensive and/or less efficient. Another notable effect is the cyclic torque loading on the generator (and any gearbox) caused by the changing angle of attack (and resulting aerodynamic loadings) during each revolution of the rotor [6]. As a result, the generator has to cope with a significantly larger peak torque loading than its HAWT equivalent. The decision has to be taken how much of this variation is the generator designed to cope with, and how large will the variations in rotational speed be.

This paper looks into the effects of cyclic torque loading on the generator, some control strategies to deal with this variable torque and the resulting effect on the generator losses and the cost of the active material of the generator.

\section{Methodology}

The testing of this case study involved defining a sample mechanical torque loading of the VAWT as well as the electrical torque response of the generator, to use with a generator model to calculate the costs and losses associated with various torque control strategies.

\subsection{Cyclic Mechanical Torque}

The cyclic loading on the generator is caused by the variation in aerodynamic loading due to the changing angle of attack on the rotor blades. As this is a periodic process it can be represented a Fourier series of sine waves. For this case study, it is simplified to a single sine wave about a mean torque as described in Equation (1).

$$
T=\bar{T}+T_{\Delta} \sin (b \theta)
$$


For each wind speed, the mechanical torque $T$ comprises the mean torque $\bar{T}$ (the torque loading of an equivalent HAWT at the same power output and rotational speed, for the same rotor radius and power coefficient $c_{p}$ ) as well as a sine wave component with a peak variation of $T_{\Delta}$ (assumed to be $50 \%$ of $\bar{T}$ for this case study) at a frequency equal to the frequency of rotation $\omega$ multiplied by the number of blades $b$.

\subsection{Electrical Torque Response: Variable Torque and Variable Speed Strategies}

The cyclic mechanical torque loading on the generator can potentially mean that the mechanical torque and the electrical torque of the generator are out of balance. The response of the rotating inertia to a torque imbalance is defined by Newton's $2^{\text {nd }}$ Law for a rotating system (Equation (2)), where $J$ is the moment of inertia of the wind turbine's rotor, driveshaft and the direct-drive generator rotor while $\alpha$ is the angular acceleration of the rotor caused by the torque imbalance, resulting in a variation in rotational speed $\omega$.

$$
T_{\text {mech }}-T_{\text {elec }}=j \alpha
$$

The electrical torque response of the generator (which is controlled by adjusting the current through the stator coils) will have a similar form to the mechanical torque in Equation (1). However there is an option to limit the variation of the electrical torque in order to limit the peak electrical torque experience by the generator. This limiting parameter is represented here as $q$ and is defined in Equation (3) as the ratio of the electrical torque variation divided by the mechanical torque variation

$$
q=\frac{T_{\Delta_{\text {elec }}}}{T_{\Delta_{\text {mech }}}}
$$

The electrical torque profiles and the resulting change in rotor speed for different $q$ strategies are demonstrated in Figure 1.

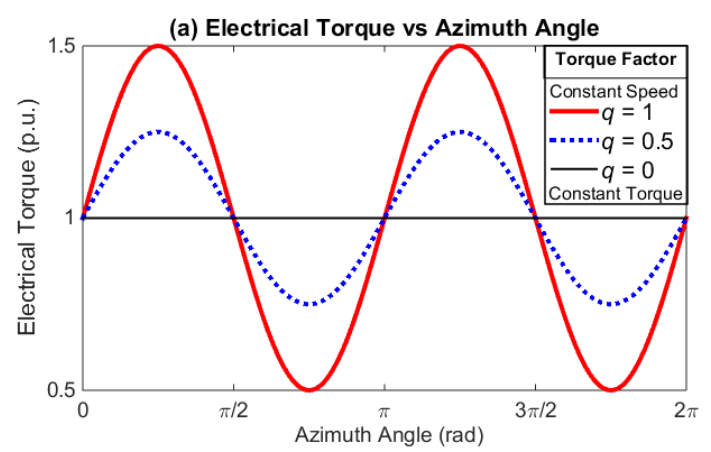

(b) Rotor Speed vs Azimuth Angle

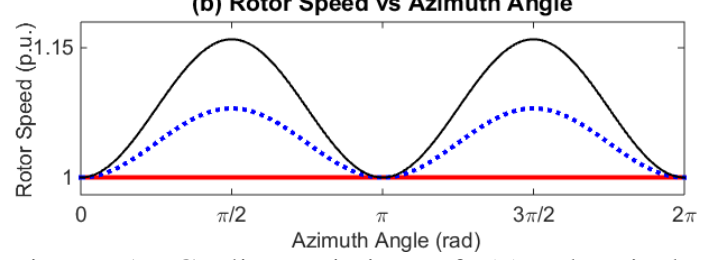

Figure 1: Cyclic variation of (a) Electrical Torque and (b) Rotor Speed for different Torque Factors $q$
When deciding the appropriate torque control strategy, there are two basic strategies (at either ends of the range for $q$ ): fixed torque operation and fixed speed operation. For Fixed Torque operation $(q=0)$ the electrical torque is kept fixed at $\bar{T}$ while the mechanical torque varies through the cycle. This setting leads to a flat electrical torque output but results in the maximum variation in rotational speed. By contrast, Fixed Speed operation $(q=1)$ is achieved when the electrical torque equals the mechanical torque at all times. The rotor speed remains constant, but this leads to the largest peak torque value of any strategy. Any $q$ setting between 0 and 1 will result in some measure of variation in both electrical torque and rotational speed; Figure 1 also shows the resulting variation for $q=0.5$.

\subsection{Generator Model}

For this case study a permanent magnet generator was simulated by modelling a generator segment (single pole pair) using a combination of an equivalent electrical circuit (as described by Polinder [9]) which is modelled in MATLAB and a magnetic circuit modelled in a Finite Element Analysis package FEMM. Both the equivalent circuit and the generator segment models are shown in Figure 2.

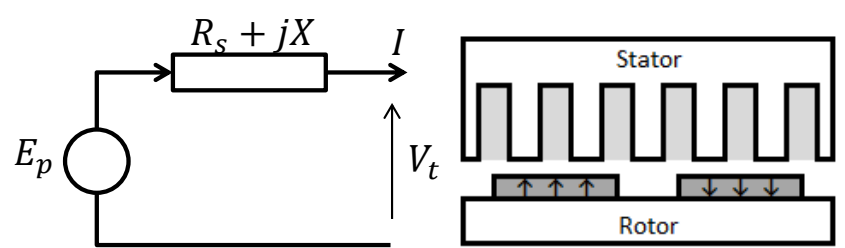

Figure 2: Equivalent Circuit and Generator Segment Models

For the given generator dimensions, FEMM calculates the flux density waveform in the airgap. This is passed to the equivalent circuit to calculate the no-load voltage. The stator resistance and magnetising inductance is modelled using the approach as outlined by Polinder [9] while the current is set to provide the desired electrical torque response.

\subsection{Generator Cost}

For this case study, the cost of the active materials in the generator are worked out by calculating the mass of the copper (stator coils), steel (stator yoke, stator teeth, rotor yoke) and permanent magnets (rotor poles) and multiplying by a unit cost of $€ 15 / \mathrm{kg}$ for copper, $€ 3 / \mathrm{kg}$ for steel and $€ 25 / \mathrm{kg}$ for permanent magnets (as used by Polinder [9]). The cost of the structural mass of the generator has not been calculated.

\subsection{Generator Losses}

This case study focused on the generator losses from the copper and the iron. The copper losses depend on the current through the stator coils which is proportional to the electrical torque response. Therefore the r.m.s. stator current can also be modelled as a sum of a mean r.m.s. current $\bar{I}$ and a sine wave component of the current with magnitude equal to $q I_{\Delta}$ 
(where $I_{\Delta}$ is the maximum r.m.s. current variation which corresponds to the maximum torque variation $T_{\Delta}$. The copper losses $P_{\mathrm{Cu}}$ are calculated using Equation (4) by integrating the varying $I^{2} R$ losses over one complete revolution of the rotor.

This equation shows that copper losses vary with the square of $q$ and the peak copper loss comes from the $q=1$ setting.

$$
P_{\mathrm{Cu}}=R\left(\bar{I}^{2}+\frac{\left(q I_{\Delta}\right)^{2}}{2}\right)
$$

The iron losses depend on the electrical frequency (determined by the number of pole pairs $p$ and the rotational speed of the rotor) and the flux density in the stator iron. The variation in electrical frequency is calculated from rearranging Equation (2) and placing into the angular equations of motion. This is then integrated over one complete revolution to calculate both the mean electrical frequency (Equation (5a)) and the mean of the square of electrical frequency (Equation (5b))

$$
\begin{aligned}
& \overline{f_{e}}=\frac{p}{2 \pi}\left(\omega_{0}+\frac{T_{\Delta}}{b J \omega_{0}}(1-q)\right) \\
& \overline{f_{e}^{2}}=\frac{p^{2}}{4 \pi^{2}}\left(\omega_{0}^{2}+\frac{2 T_{\Delta}}{b J}(1-q)\right)
\end{aligned}
$$

The iron losses $P_{\mathrm{Fe}}$ are calculated by first separately calculating the specific losses (per unit mass) for the stator yoke and the stator teeth using Equation (14) from Polinder [9] and multiplying by the mass of each steel segment $i$ (where $\widehat{B}_{F e}$ is the flux density though the specific segment). The final equation for iron loss is given in Equation (6a) with the coefficients $A_{\mathrm{h}}$ and $A_{\mathrm{e}}$ (as described by Polinder) listed in Equation (6b).

$$
\begin{gathered}
P_{\mathrm{Fe}}=\sum_{\mathrm{i}}\left(A_{h} \overline{f_{e}}+A_{e} \overline{f_{e}^{2}}\right) \hat{B}_{F e_{\mathrm{i}}}{ }^{2} m_{\mathrm{i}} \\
A_{\mathrm{h}}=\frac{2 P_{F e \mathrm{~h}}}{f_{0} \hat{B}_{0}^{2}} \quad A_{e}=\frac{2 P_{F e 0 \theta}}{f_{0}{ }^{2} \hat{B}_{0}{ }^{2}}
\end{gathered}
$$

The iron losses are reduced by decreasing the mean electrical frequency which (as seen in Figure 1b) occurs for the fixed speed $q=1$ setting. Note that both the copper and iron losses are average power losses across each revolution.

\subsection{Case Study Generator Specifications}

The generator used for this case study is a 5MW directlydriven permanent magnet generator for use in an H-rotor VAWT for offshore generation. It has evolved from Polinder's 3MW generator for a HAWT [9] to a VAWT generator (based on parameters from Michon [3] including power output and rotational speed). The generator has a stator radius of $5.6 \mathrm{~m}$, a stack length of $2.6 \mathrm{~m}$ and it is comprised of 160 pole pairs. More specifications are listed in the appendix at the end of this paper.

\section{Results}

The following tests are carried out using a fixed generator segment.

\subsection{Generator Losses for a Fixed Wind Speed}

The first set of results in Figure 3 demonstrate how the copper and iron losses vary for different torque factor $q$ settings (for a single fixed wind speed of $9 \mathrm{~m} / \mathrm{s}$ ).

The copper losses increase with the square of $q$ (as in Equation (4)), while the iron losses decrease linearly with $q$ (Equations (5)-(6)). For the test turbine at this wind speed, the two losses are of similar magnitude. In general the relative magnitudes of these losses will vary with $q$. At the wind speed of $9 \mathrm{~m} / \mathrm{s}$ the total loss of the generator is minimised when the torque factor is set to $q=0.4$.

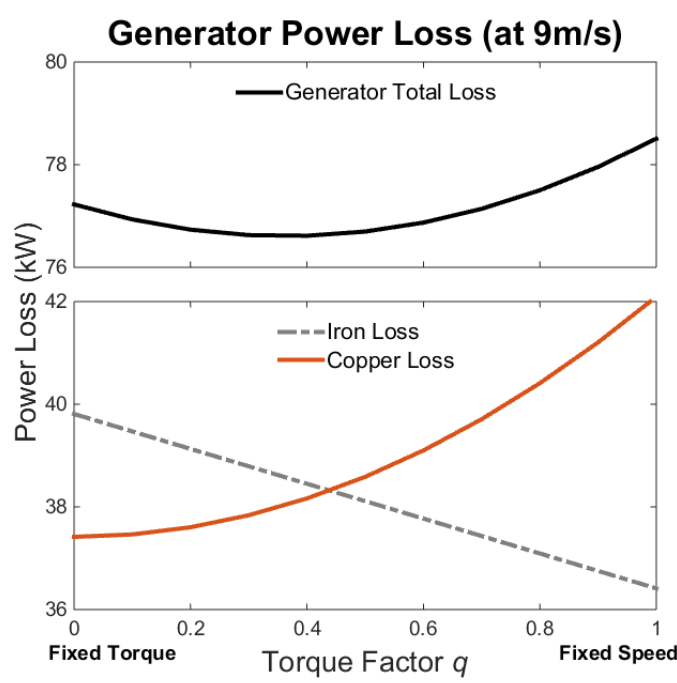

Figure 3: Generator Losses (at $9 \mathrm{~m} / \mathrm{s}$ wind speed)

\subsection{Strategies to Minimise Generator Losses}

The next stage involves assessing the combined generator loss for different $q$ strategies across the full range of wind speeds. In the interests of clarity the results presented on Figure 4 show the difference in the generator loss compared to the baseline fixed torque strategy of $q=0$.

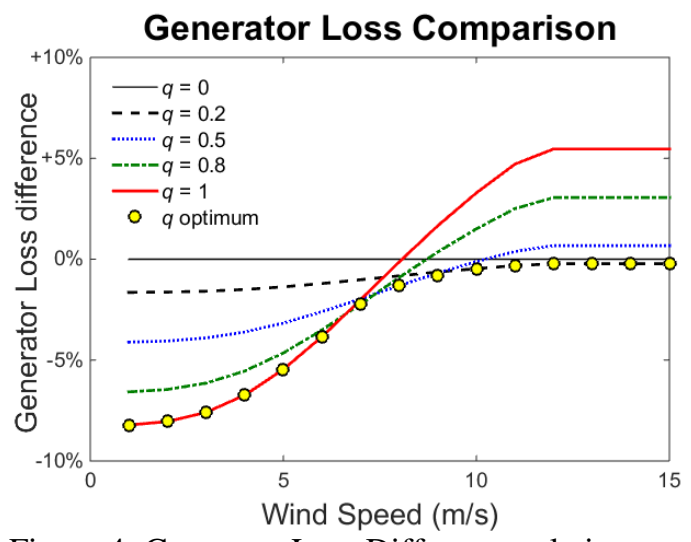

Figure 4: Generator Loss Difference relative to $q=0$ baseline 
For each wind speed, the torque factor $q$ that minimised the total generator losses was selected as part of the optimum- $q$ strategy for loss minimisation. This strategy is described in Figure 5 which results in fixed speed operation $(q=1)$ for lower wind speeds with $q$ reducing as the wind speed approaches the rated speed of the turbine before settling at a final setting of $q=0.2$ for rated power operation.

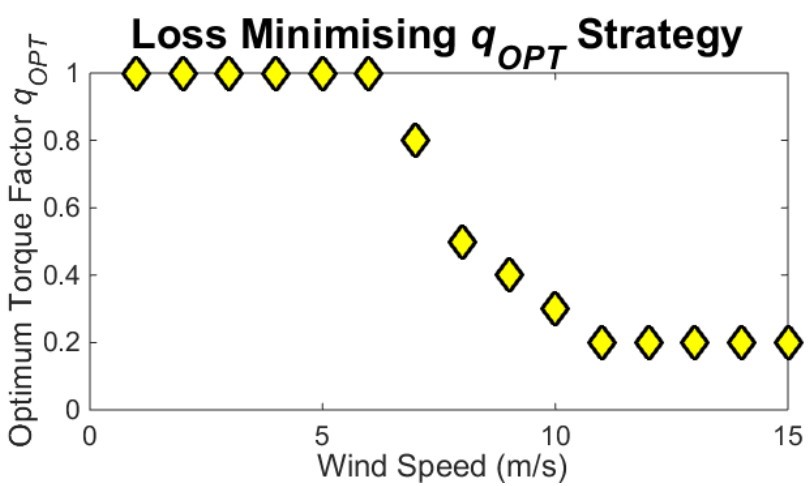

Figure 5: $q$ Strategy to Minimise Generator Losses

The fact that $q_{\mathrm{OPT}}$ does not trend to 0 (fixed torque operation) required some investigation. Therefore the copper and iron losses at a wind speed above rated (e.g. $15 \mathrm{~m} / \mathrm{s}$ ) were investigated in Figure 6. For this turbine at low $q$ settings, the quadratic increase in copper losses is initially slower than the linear decrease in iron losses; therefore the total generator losses decrease until the optimal point at around $q=0.2$.

\section{Generator Power Loss (at $15 \mathrm{~m} / \mathrm{s}$ )}

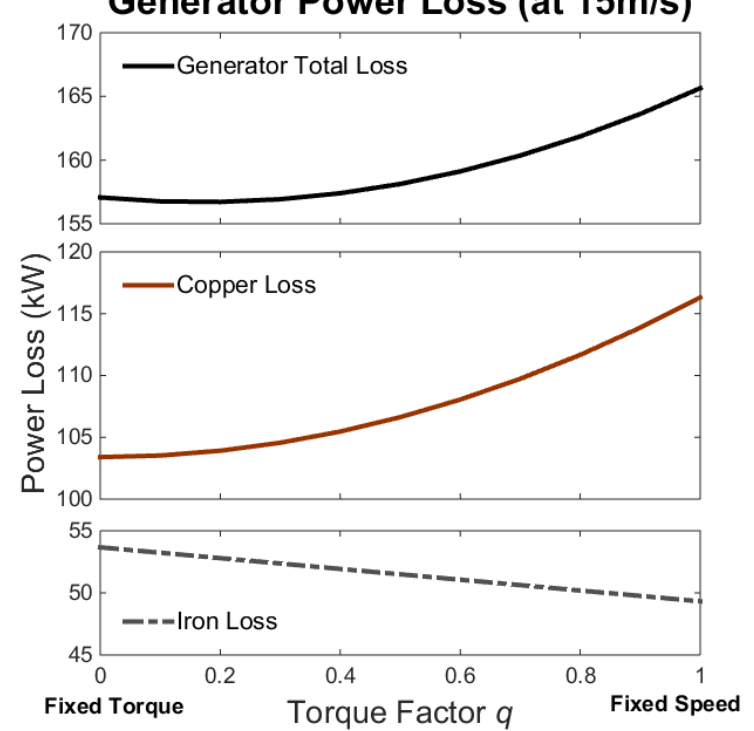

Figure 6: Generator Losses (at $15 \mathrm{~m} / \mathrm{s}$ wind speed)

\subsection{Annual Energy Losses from Generator}

Following up from the generator power loss calculations in Figure 4 , the annual energy losses for each $q$ strategy is calculated using the Weibull distribution to calculate the number of hours per year that each wind speed is experienced, multiplying this by the energy loss per hour at each wind speed and summing up for all wind speeds. These results, as well as comparison against the fixed torque $q=0$ baseline strategy is shown in Table 1.

\begin{tabular}{|l|l|l|}
\hline Torque Factor $q$ & Annual Losses $(M W h)$ & $\%$ Loss (vs q=0) \\
\hline 0 & 508.0 & $0.0 \%$ \\
0.2 & 504.8 & $-0.6 \%$ \\
0.4 & 504.1 & $-0.8 \%$ \\
0.6 & 505.9 & $-0.4 \%$ \\
0.8 & 510.1 & $+0.4 \%$ \\
1 & 516.9 & $+1.8 \%$ \\
Optimum- $q$ & 500.4 & $-1.5 \%$ \\
\hline
\end{tabular}

Table 1: Annual Energy Losses for different $q$ strategies

For this turbine, the best fixed $q$ strategy is to set the torque factor to $q=0.4$, resulting in a $0.8 \%$ loss reduction against the baseline fixed torque $(q=0)$ strategy. In contrast the strategy that maximises losses is the fixed speed $(q=1)$ strategy which sees a $1.8 \%$ increase over the baseline.

For comparison the loss minimisation optimum- $q$ strategy reduced losses by an additional $0.7 \%$ reduction in losses over the best fixed $q$ strategy.

\subsection{Generator Torque Loading}

The test generator is designed to cope with the peak electrical torque loading experienced at rated power from the $q=1$ strategy. The other $q$ strategies have a lower peak electrical torque as demonstrated in Figure 7.

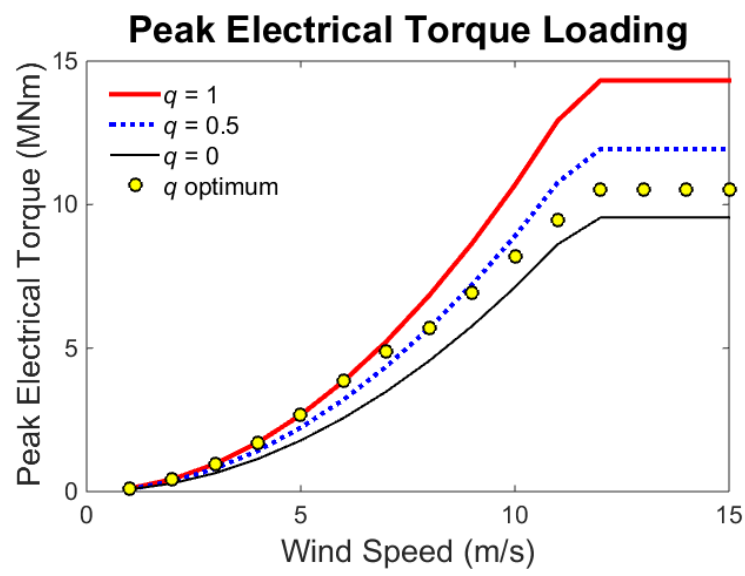

Figure 7: Peak Electrical Torque at different wind speeds for different $q$ strategies

An important result to note is that the optimum- $q$ strategy (that minimises the generator losses at each wind speed) results in a significantly lower peak torque experienced than the $q=1$ setting that the generator is designed to handle (in this case the peak torque is equal to that of the $q=0.2$ fixed strategy). It is therefore envisaged that the generator can be designed to handle a lower peak torque than the $q=1$ peak.

\subsection{Generator costs}

One of the parameters that determines the sizing of the generator is the peak torque that it is expected to deal with. In 
this spirit, the parameter $q_{\text {MAX }}$ is defined as the setting for $q$ for which (at rated wind speed $v_{\text {rated }}$ ) the corresponding electrical torque is equal to the torque rating of the generator $T_{\text {rating. }}$. The relationship between $q_{\text {Max }}$ and $T_{\text {rating }}$ is described by Equation (7).

$$
T_{\text {rating }}=\bar{T}\left(v_{\text {rated }}\right)+q_{\text {MAX }} T_{\Delta}\left(v_{\text {rated }}\right)
$$

This is a parameter based on the design of the generator, and as such running a $q$ setting greater than $q_{\text {Max }}$ would not be allowed at rated operation (as the electrical torque would exceed the rating of the machine), although running a high $q$ setting at lower wind speeds would be possible so long as the peak torque does not exceed $T_{\text {rating }}$ (see Figure 7).

Up to this point, the test generator has been scaled to $q_{\text {Max }}=1$ which allows the generator to handle any $q$ strategy between fixed torque and fixed speed. For this test, the generator is rescaled by reducing the number of generator segments which reduce the number of pole pairs and thus the radius of the generator rotor (the stack length is constant).

The costs of the active materials of the generator (copper, steel and permanent magnets) are shown in Table 2 which demonstrates the relationship between the allowed electrical torque variation and the cost of the generator.

\begin{tabular}{|l|l|l|l|}
\hline $\begin{array}{l}\text { Max Torque } \\
\text { Factor } q_{\text {MAX }}\end{array}$ & $\begin{array}{l}\text { Pole } \\
\text { Pairs }\end{array}$ & $\begin{array}{l}\text { Generator } \\
\text { Active Cost }(\text { kEuro })\end{array}$ & $\begin{array}{l}\text { \% Cost } \\
\left.\text { (vs } q_{\text {MAX }}=1\right)\end{array}$ \\
\hline 1 & 160 & 765.3 & $0.0 \%$ \\
0.8 & 155 & 739.1 & $-3.4 \%$ \\
0.6 & 149 & 712.4 & $-6.9 \%$ \\
0.4 & 144 & 684.0 & $-10.6 \%$ \\
0.2 & 138 & 654.8 & $-14.4 \%$ \\
0 & 131 & 624.6 & $-18.4 \%$ \\
\hline
\end{tabular}

Table 2: Cost of Active Materials for different $q_{\text {Max }}$ designs

\section{Discussion}

\subsection{How $q$ affects copper and iron losses}

The copper losses increase when the peak current is increased which occurs when the current, and equivalently the electrical torque, have a higher variation, i.e. high $q$ (see Equation (4)). This is because of the $I^{2} R$ losses which for larger current variations increase overall due to the increase in losses at peak current being much larger than the decrease in losses at the minimum current value.

Conversely the iron losses increase with higher electrical frequencies which occur with smaller variations in electrical torque (i.e. low $q$ ). These calculations (Equations (5)-(6)) are more complex as they depend on the both the mean electrical frequency and the mean of the square of electrical frequency. Both quantities have a term that depends linearly on $(1-q)$, thus the iron losses decrease linearly with $q$ due in particular to the reduction of mean $f_{\mathrm{e}}^{2}$ at higher $q$ when the speed variation (and thus maximum speed) reduces.
The relative magnitude of these losses varies depends on the wind speed as well as the design of the generator. At high wind speeds, the copper losses are more significant than the iron losses because of the increased current to supply the larger electrical torque. Iron losses are relatively more significant at lower wind speeds due to the decreased copper losses from the reduced current.

\subsection{Optimal $q$ at different wind speeds}

The optimal- $q$ strategy sets the torque factor $q$ to the value that minimises the generator losses at each individual wind speed. At lower wind speeds this is towards $q=1$ (fixed speed at wind speed, $v$ ) due to the priority to minimise iron losses. At higher wind speeds this tends toward $q=0$ (fixed torque at wind speed, $v$ ) due to the priority to minimise copper losses.

In between these two extremes, there is a cross-over region where the rate of increase in copper losses is of a similar magnitude to the rate of decrease in iron losses. Here the optimal $q$ is in-between the fixed torque and fixed speed extremes. The location of the cross-over varies for different generator designs (for this turbine it is at around $9 \mathrm{~m} / \mathrm{s}$ ), but the important principle is to apply the separate relationships for copper loss and iron loss, and evaluate based on the specific generator design how these balance to reduce the overall generator losses.

\subsection{Resizing Generator to Reduce Costs}

As demonstrated in Figure 7 the peak electrical torque for all fixed $q$ strategies, as well as the optimum- $q$ strategy, occurs at rated operation. The larger the peak torque requirement, the larger the generator has to be to spread out this electrical torque loading across the active area of the generator (effectively the airgap between the rotor and stator). This leads to an increased mass and thus an increased cost.

An important consequence of the optimum- $q$ strategy is that the peak torque of this strategy is the same as that for the $q=0.2$ strategy. Therefore if the baseline generator $(q=1)$ is running the optimum- $q$ strategy then it is under-utilised (it was rated at $15 \mathrm{MNm}$ but only runs at a peak of $11 \mathrm{MNm}$ ). With this fact, it would be possible to reduce the size of the generator, to that designed to cope with a lower peak torque, resulting in a generator cost reduction of around 10-15\% provided that the generator is controlled to a maximum $q$ of between 0.2-0.4 at near-rated and rated wind speeds. This resizing of the generator would result in changes to the copper losses (generally $I^{2} R$ losses will increase to compensate for a lower induced emf) and iron losses (generally smaller as there is less iron mass and also there is a lower frequency due to reduced pole number) due to the resizing.

A generator that allows no electrical torque variation at rated speed $\left(q_{\text {Max }}=0\right)$ results in a reduction in active cost of $18 \%$, however the generator would have no method of using the generator to control the speed of the turbine at rated power. 


\subsection{Generator design for optimal $q$}

It is likely that the optimal generator design for a VAWT will have a different balance of copper and iron losses to that of an equivalent HAWT. Although this baseline generator design is not optimised and is just used here as a case study it shows some features that are useful. With relatively small copper losses and relatively high iron losses at lower wind speeds, a high value of $q$ at lower wind speeds can be accommodated without leading to extra generator cost. At higher wind speeds where the copper losses dominate and so $q \rightarrow 0$, meaning that extra generator cost is modest. Machines with higher iron loss density at rated wind speed would give higher optimal $q$ implying extra generator cost.

\subsection{Future Research}

Future work to be carried out in this area will investigate the effect on the aerodynamic efficiency of the turbine rotor blades that result from a fixed torque/variable speed strategy from the generator. It may be that the variable speed through a rotation leads to aerodynamic losses which outweigh the generator copper and iron losses.

In addition, the effect on the copper and iron losses from rescaling the turbine to a different $q_{\text {Max }}$ will be investigated. In particular whether the change in losses from rescaling is separable (either in absolute or relative losses) from the torque factor $q$ setting of the generator, thus allowing this two factors to be independently calculated and applied to the overall effect on copper and iron losses. The result of this work will allow the generator design and the turbine/generator strategy to be optimised to minimise the cost of energy.

\section{Conclusions}

This case study shows that adjusting the torque/speed control strategy of the generator can lead to some potential generator loss reductions and allows the generator to adapt to changing wind speeds and can lead to the biggest potential savings. In general, running a fixed speed strategy minimises losses at low wind speeds, while electrical torque variation should be reduced at higher wind speeds. The optimal setting for each wind speed will depend on how the copper and iron losses balance which depends on the design of that turbine's generator. In addition this loss-minimisation strategy (with different $q$ settings for different wind speeds) results in the reduction in the peak electrical torque experience by the generator, which allows the opportunity to use a smaller, cheaper generator designed to that lower torque rating (as opposed to the full variation peak torque).

There are other factors that need to be investigated and factored in to the generator model in order to progress with the overall aim of optimising the design of the generator and drivetrain for operation in an offshore VAWT. When the optimisation (factoring in all the knock-on effects of resizing the generator) is carried out, the cost of energy of the VAWT with the optimal generator design will be compared with that of conventional HAWTs to assess if VAWTs might be viable for commercial offshore generation.

\section{Acknowledgements}

This research was funded by the EPSRC through the Centre for Doctoral Training in Wind Energy Systems at the University of Strathclyde, award no. EP/G037728/1.

\section{References}

[1] M. Barone and J. Paquette, "Vertical-Axis Wind Turbines Revisited: A Sandia Perspective," Sandia Natl. Lab. 2012 Wind Turbine Bl. Work., 2012.

[2] H. J. Sutherland, D. E. Berg, and T. D. Ashwill, “A Retrospective of VAWT Technology," 2012.

[3] M. Michon, "Permanent Magnet Machines for Vertical Axis Wind Turbines," in UK Magnetics Society Meeting "Permanent Magnets - Cradle to Grave”, Stoke on Trent, 2012.

[4] VertAx Wind Ltd, "Vertical Axis Wind Turbines," 2009. [Online]. Available: http://vertaxwind.com/. [Accessed: 14-Oct-2015].

[5] European Wind Energy Association, “Aiming High Rewarding Ambition in Wind Energy," 2015.

[6] S. Eriksson, H. Bernhoff, and M. Leijon, "Evaluation of different turbine concepts for wind power," Renew. Sustain. Energy Rev., vol. 12, no. 5, pp. 1419-1434, 2008.

[7] S. Eriksson, "Direct Driven Generators for Vertical Axis Wind Turbines," Uppsala University, Sweden, 2008.

[8] T. J. Price, "UK Large-scale Wind Power Programme from 1970 to 1990: the Carmarthen Bay experiments and the Musgrove Vertical-Axis Turbines," vol. 44, no. 0, 2006.

[9] H. Polinder, F. F. a Van Der Pijl, G. J. De Vilder, and P. J. Tavner, "Comparison of direct-drive and geared generator concepts for wind turbines," IEEE Trans. Energy Convers., vol. 21, no. 3, pp. 725-733, 2006.

\section{Appendix: Generator Data}

\begin{tabular}{|l|l|}
\hline Rated Power & $5 \mathrm{MW}$ \\
\hline Rated Speed & $12 \mathrm{~m} / \mathrm{s}(@ 5 \mathrm{rpm})$ \\
\hline Turbine System Moment of Inertia & $1.05 \times 10^{8} \mathrm{~kg} \mathrm{~m}^{2}$ \\
\hline Stator Radius & $5.6 \mathrm{~m}$ \\
\hline Stack Length & $2.6 \mathrm{~m}$ \\
\hline Pole Pairs / Pole Pitch & $160 / 110 \mathrm{~mm}$ \\
\hline Stator Tooth (Width $\times$ Height) & $18 \mathrm{~mm} \times 80 \mathrm{~mm}$ \\
\hline Stator Slot (Width $\times$ Height) & $18.6 \mathrm{~mm} \times 80 \mathrm{~mm}$ \\
\hline Stator Yoke (Height) & $40 \mathrm{~mm}$ \\
\hline Rotor Yoke (Height) & $40 \mathrm{~mm}$ \\
\hline Rotor Magnet (Width $\times$ Height) & $79 \mathrm{~mm} \times 15 \mathrm{~mm}$ \\
\hline Air Gap & $5 \mathrm{~mm}$ \\
\hline
\end{tabular}

Table 3: Case Study Generator Data $\left(q_{\text {Max }}=1\right)$ 